
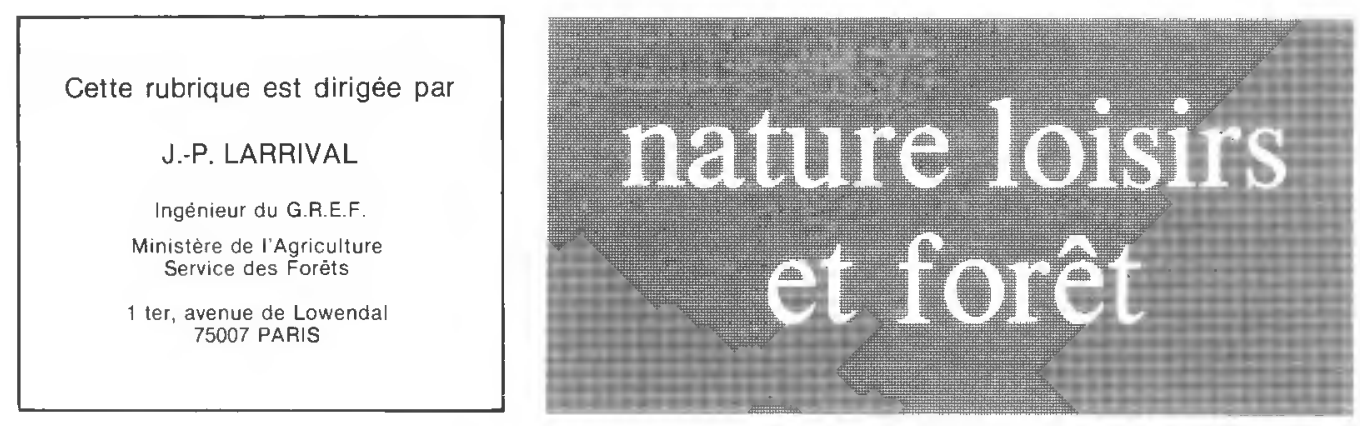

\title{
L'ACCUEIL DU PUBLIC EN FORÊT LES CENTRES D'INTERPRÉTATION DE LA NATURE AU QUÉBEC
}

\author{
E. DE GUILLEBON
}

Lors d'un voyage d'études au Québec en août 1977, certains élèves de l'École nationale du génie rural, des eaux et des forêts ont visité des centres d'interprétation de la nature et parcouru des sentiers de nature dépendant du ministère québecois des Terres et Forêts. II est intéressant de rapporter cette expérience et d'étudier les possibilités de telles réalisations en France.

\section{LA NATURE, CELA S'APPREND}

Le centre d'interprétation de la nature vise à promouvoir l'éducation du public en matière de conservation de la nature, grâce à un aménagement léger permettant l'accessibilité du territoire. Le service de l'Éducation en conservation du ministère des Terres et Forêts du Québec met à profit tous les éléments de l'environnement susceptibles de faire ressortir les liens qui unissent les êtres vivants et leur milieu naturel. Le centre offre aux visiteurs différentes activités éducatives, culturelles et scientifiques leur permettant de découvrir et d'apprendre à respecter la nature, de comprendre les actions des gestionnaires. Le centre et les sentiers sont les deux moyens de cette politique, dans un pays où la nature n'était jusqu'ici perçue que comme lieu de promenade en voiture, ou comme territoire de chasse ou de pêche.

Le centre comporte un bâtiment regroupant des salles d'exposition, des salles de conférences, des laboratoires, une bibliothèque et parfois le logement des naturalistes qui y travaillent.

Des expositions permettent de focaliser l'attention des visiteurs sur certains points particuliers qu'ils pourront observer à l'extérieur. A Duchesnav - le centre est inclus dans la station fores- 


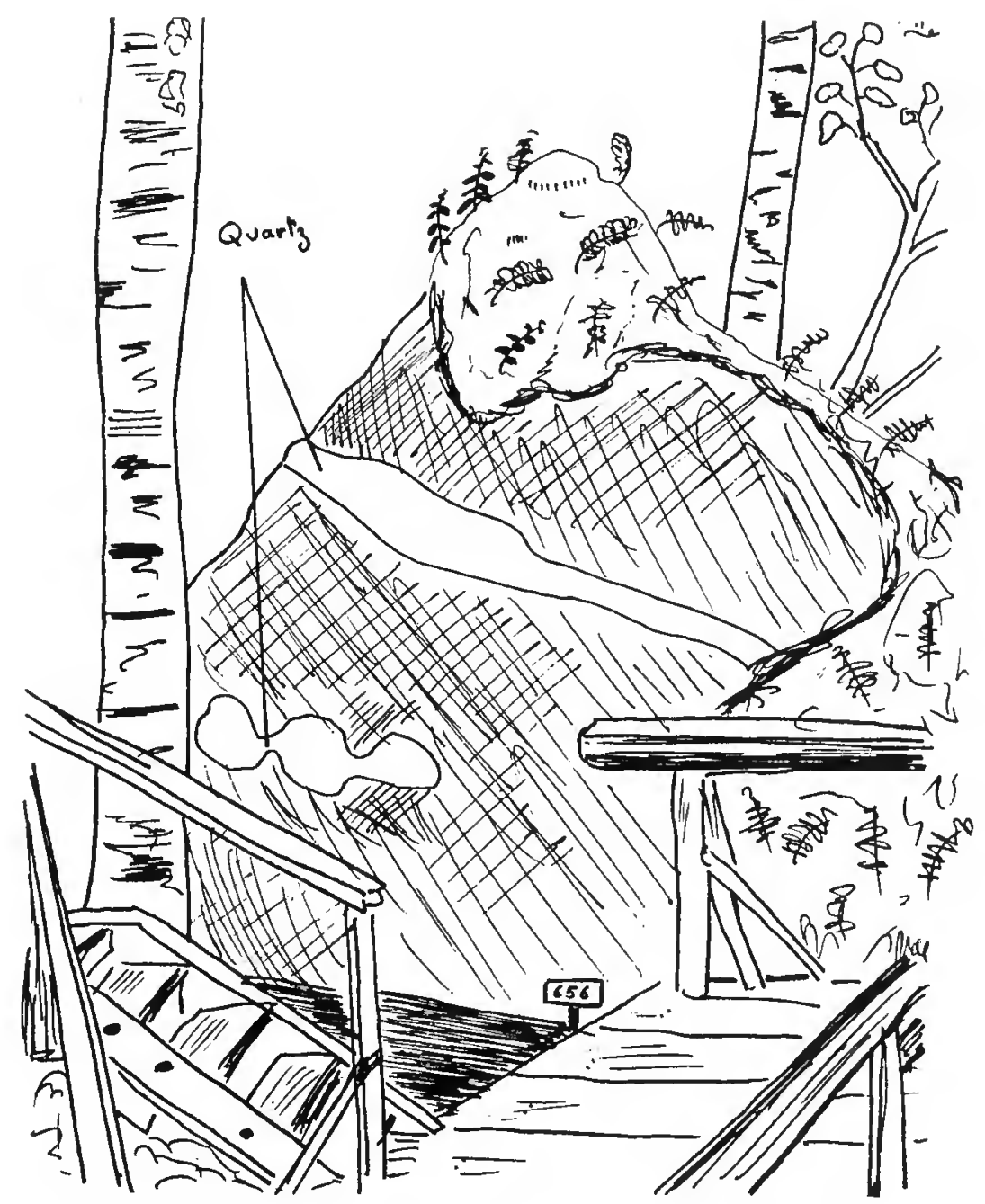

Station $\mathbf{6 5 6}$

Le quartz, un minéral trés dur.

tière de recherche à 50 kilomètres de Québec - étaient disposés sur des panneaux des fragments de troncs accompagnés de silhouettes d'arbres qu'il fallait identifier. D'autres panneaux illustraient le thème de l'eau : cycle de l'eau, vie aquatique, végétation des marais, mœurs des castors, dégradations causées par l'homme. Ailleurs, on expliquait la formation du balai de sorcière. Ces expositions peuvent être visitées seul ou accompagné de naturalistes. Ces jeunes gens et jeunes filles, techniciens ou étudiants en écologie, zoologie, botanique ou foresterie ont un contrat de six mois avec le ministère.

De plus, il est possible pour les commissions scolaires du Québec de profiter à peu de frais de toutes les facilités nécessaires à la tenue de classes de nature ou classes vertes, pendant l'année scolaire. On initie de cette façon les enfants à la nature, ce qui est la meilleure façon d'atteindre les adultes. Le bricolage et la création artistique à partir de matériaux ramassés en forêt sont très appréciés.

Les sentiers sont l'élément fondamental des centres d'interprétation de la nature. II s'agit de parcours en forêt, le long des lacs ou sur des collines, établis de telle sorte que le visiteur qui les suit puisse y trouver un intérêt. Ces parcours ne sont jamais longs. La marche n'est pas 
répandue au Québec et il ne faudrait pas rebuter le citadin qui ne connaît la nature que par les vitres de son auto. Les sentiers sont matérialisés sur le terrain par des cailloux, des passages sablés, des rondins, des trottoirs en bois; ils empruntent des escaliers, des passerelles, et présentent des stations repérées par un numéro peint sur un écriteau.

Un livre décrivant toutes les stations a été distribué à l'accueil. Par exemple, la station 290 du sentier Sabot au Centre des Pallissades permet d'observer des pins rouges (Pinus resinosa), des pins blancs (Pinus strobus) et des pins gris (Pinus banksiana). Le livret Sabot explique, à la station 290 , les différences entre ces pins. Le numéro de la station correspond à sa distance en mètres du départ (nous sommes dans un pays qui se met au système métrique).

Le livret reste la propriété du promeneur qui pourra s'y reporter plus tard quand il retrouvera des pins, s'il n'a pas retenu la leçon. Cette méthode est très éducative : le promeneur ne lit plus passivement de vagués inscriptions clouées sur les arbres; tout l'incite à ouvrir le livret et à faire l'effort de lire ce qui se rapporte à ce qu'il voit. Peu à peu, il apprend à regarder et même à comprendre. Les inscriptions sur le terrain sont inexistantes, nonobstant les noms de quelques plantes : cornouiller canadien, clintonie boréale, etc., qui reviennent sans cesse. La visite des sentiers peut être, à la demande, guidẻe par un naturaliste qui répond à toutes les questions des nromeneurs tout en les empêchant de commettre des dégâts.

Avant de s'engager dans les sentiers, les promeneurs sont invités à se procurer au centre d'accueil des sacs de matière plastique destinés à recueillir les papiers, récipients vides et autres rebuts. Au retour, ils n'auront qu'à les jeter dans les poubelles mises à leur disposition. Ceci évite d'entretenir des poubelles partout et apprend au public á transporter ses déchets. II n'y aura pas de poubelles quand il ira se promener dans la "forêt sauvage"! Le réflexe de garder ses déchets commence à être acquis.

Les feux de tous ordres sont prohibés et il est interdit de fumer en forêt. Il est demandé aux visiteurs de ne pas casser d'arbres, ni d'âbimer le matériel, ni de marcher hors des sentiers. Mais les naturalistes n'ont pas de pouvoir de police et la persuasion n'est pas toujours efficace. Certains parcs de nature dépendant du Gouvernement fédéral ont des gardes.

Installation d'accueil aux Palissades.

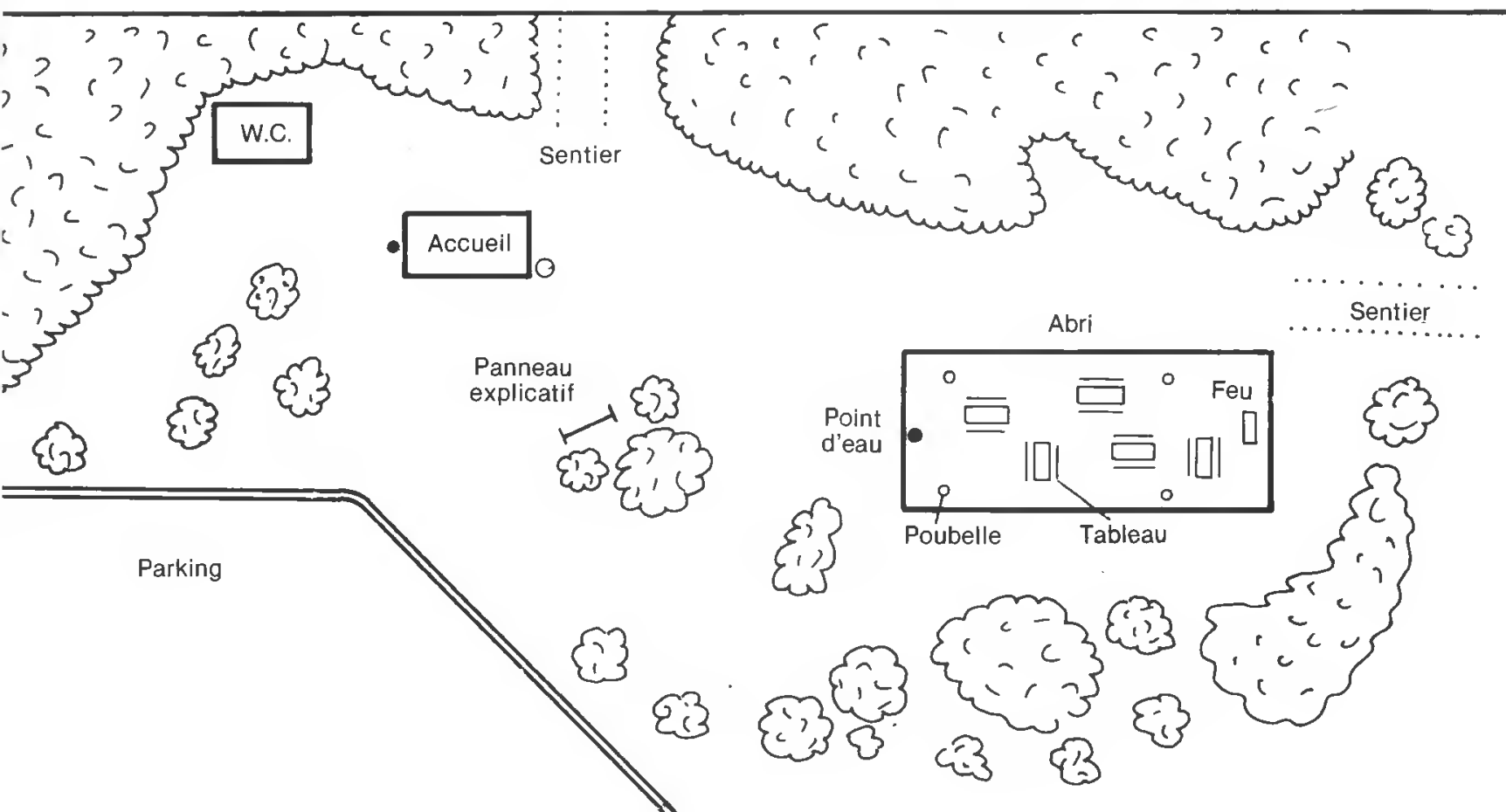




\section{E. DE GUILLEBON}

\section{CRITIQUE}

Les centres d'interprétation de la nature semblent être efficaces. D'après le personnel qui y travaille, la notion de conservation de la nature commence à naître dans un pays où tout semblait donné pour rien et à profusion. Il est donc payant d'entreprendre de tels eflorts.

Le sentier apparaît très contraignant pour des Européens : son tracé et son balisage sont très artificiels et certains aménagements sont trop lourds. On a reproché à un de nos élèves de ne pas remettre à sa place un caillou qu'il avait soulevé : "Ce pourrait être une demeure à fourmis... ". Mais il faut comprendre que ces centres sont destinés à un vaste public qui n'a pas l'habitude de la forêt et qu'ils sont de plus en plus visités (200 à 1000 visiteurs par dimanche). Ces centres sont la première étape dans la connaissance du milieu et doivent donner au public le goût d'aller en forêt. La prochaine étape sera sans doute l'ouverture de sentiers de randonnée pédestre.

\section{EN FRANCE}

A l'inverse des Québecois, de nombreux Français ont l'habitude d'aller en forêt, et ils sont très attachés à ces promenades. Mais chacun sait qu'ils connaissent mal et cette forêt, et l'action des forestiers. On peut alors penser aux sentiers d'initiation.

Ces sentiers seraient des parcours de 3 ou 4 kilomètres en milieu boisé, parsemés de stations. Pas besoin d'un balisage compliqué; un sentier est vite tracé : la litière enlevée, des branches gênantes coupées et des cailloux ou des pierres pour indiquer le chemin, parfois un pont rustique. Ceci pourrait être installé par des chantiers de jeunes ou d'étudiants ou par des clubs de nature sous la surveillance des services forestiers. Un livret, analogue à ceux qui étaient autrefois édités par les Eaux et Forêts sur de nombreuses grandes forêts françaises, distribué dans les offices de tourisme, décrirait les stations et donnerait des conseils.

Certaines forêts très fréquentées comme Tronçais, La Joux ou Fontainebleau pourraient proposer des centres de nature et offrir les services de naturalistes ou de forestiers. Les équipements lourds et coûteux sont à bannir : ils transforment la forêt et lui donnent un visage trop urbanisé. Les centres et les sentiers respectent par leur essence même le milieu.

L'expérience d'accueil du public en forêt au Québec et très instructive et encourageante. Nos cousins d'outre-Atlantique nous ont appris à faire découvrir la nature au public ; pourquoi ne pas développer encore plus l'expérience actuelle chez nous?

EMMANUEL DE GUILLEBON

33. rue Dumez

59340 ROSENDAEL 